\title{
Geographic variability in potentially discretionary red blood cell transfusions after coronary artery bypass graft surgery
}

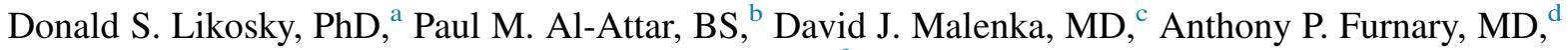 \\ Eric J. Lehr, MD, PhD, ${ }^{\text {e }}$ Gaetano Paone, MD, MHSA, ${ }^{f}$ Mallika Kommareddi, MPH, ${ }^{a}$ Robert Helm, MD, ${ }^{g}$ \\ Ruyun Jin, MD, MCR, ${ }^{h}$ Charles Maynard, PhD, ${ }^{i}$ Eric C. Hanson, MD,${ }^{j}$ Elaine M. Olmstead, BA, ${ }^{c}$ \\ Todd A. Mackenzie, $\mathrm{PhD},{ }^{\mathrm{c}}$ Cathy S. Ross, MS, ${ }^{\mathrm{c}}$ and Min Zhang, $\mathrm{PhD},{ }^{\mathrm{k}}$ for the Cardiac Surgery Quality \\ Improvement Network
}

Objective: A number of established regional quality improvement collaboratives have partnered to assess and
improve care across their regions under the umbrella of the Cardiac Surgery Quality Improvement (IMPROVE)
Network. The first effort of the IMPROVE Network has been to assess regional differences in potentially discre-
tionary transfusions $(<3$ units red blood cells [RBCs]).

Methods: We examined 11,200 patients undergoing isolated nonemergent coronary artery bypass graft surgery across 56 medical centers in 4 IMPROVE Network regions between January 2008 and June 2012. Each center submitted the most recent 200 patients who received 0,1 , or 2 units of RBC transfusion during the index admission. Patient and disease characteristics, intraoperative practices, and percentage of patients receiving RBC transfusions were collected. Region-specific transfusion rates were calculated after adjusting for pre- and intraoperative factors among region-specific centers.

Results: There were small but significant differences in patient case mix across regions. RBC transfusions of 1 or 2 units occurred among $25.2 \%$ of coronary artery bypass graft procedures $(2826$ out of 11,200$)$. Significant variation in the number of RBC units used existed across regions (no units, 74.8\% [min-max, 70.0\%-84.1\%], 1 unit, 9.7\% [min-max, 5.1\%-11.8\%], 2 units, $15.5 \%$ [min-max, 9.1\%-18.2\%]; $P<.001$ ). Variation in overall transfusion rates remained after adjustment $(9.1 \%-31.7 \% ; P<.001)$.

Conclusions: Delivery of small volumes of RBC transfusions was common, yet varied across geographic regions. These data suggest that differences in regional practice environments, including transfusion triggers and anemia management, may contribute to variability in RBC transfusion rates. (J Thorac Cardiovasc Surg 2014;148:3084-9)

See related commentary on pages 3089-91.

From the Department of Cardiac Surgery, ${ }^{a}$ University of Michigan, Ann Arbor, Mich; University of Michigan Medical School, ${ }^{\mathrm{b}}$ Ann Arbor, Mich; Departments of Medicine and The Dartmouth Institute for Health Care Policy and Clinical Practice, ${ }^{c}$ Geisel School of Medicine at Dartmouth, Hanover, NH; Starr-Wood Cardiac Group, ${ }^{\mathrm{d}}$ Portland, Ore; Swedish Heart and Vascular Institute, ${ }^{\mathrm{e}}$ Seattle, Wash; Division of Cardiac Surgery, ${ }^{\mathrm{f}}$ Department of Surgery, Henry Ford Hospital, Detroit, Mich; Department of Surgery, ${ }^{\mathrm{g}}$ Portsmouth Regional Hospital, Portsmouth, NH; Medical Data Research Center, ${ }^{\mathrm{h}}$ Providence Health and Services, Portland, Ore; Clinical Outcomes Assessment Program, ${ }^{\mathrm{i}}$ Seattle, Wash; Department of Cardiovascular Surgery, ${ }^{\mathrm{j}}$ Beaumont Hospital, Troy, Mich; and Department of Biostatistics, ${ }^{\mathrm{k}}$ University of Michigan, Ann Arbor, Mich.

This project was funded in part under National Cardiovascular Surgery Quality Improvement Network Planning Conferences (grant number 1R13HS020562-01) from the Agency for Healthcare Research and Quality (AHRQ), U.S. Department of Health and Human Services. The opinions expressed in this document are those of the authors and do not reflect the official position of AHRQ or the U.S. Department of Health and Human Services.

Disclosures: Authors have nothing to disclose with regard to commercial support. Received for publication Feb 11, 2014; revisions received June 27, 2014; accepted for publication July 28, 2014; available ahead of print Sept 13, 2014.

Address for reprints: Donald S. Likosky, PhD, Section of Health Services Research and Quality, Department of Cardiac Surgery (5346 CVC), University of Michigan Medical School, Ann Arbor, MI 48109-5864 (E-mail: likosky@umich.edu).

$0022-5223 / \$ 36.00$

Copyright $(\odot) 2014$ by The American Association for Thoracic Surgery

http://dx.doi.org/10.1016/j.jtcvs.2014.07.106
For more than 2 decades the Society of Thoracic Surgeons (STS) (and more recently, public and private payers) has encouraged cardiothoracic surgeons to participate in a national cardiac surgical registry designed to facilitate the assessment of clinical practice and outcomes from these surgical procedures. Data from this registry have been used for research, as well as to support quality assurance and improvement. Although contributing surgeons receive detailed reports describing the process and outcomes of their care, comparative outcomes reporting alone often fails to positively transform care.

Regional voluntary collaboratives for heart surgery have also emerged over the past several decades. Unlike a registry, a surgical collaborative is a consortium of institutions that discusses opportunities for working together to improve the quality and safety of care. Within cardiac surgery, such collaboratives are typically state-based with the majority using STS data elements. Many regional collaboratives have leveraged physician champions to convene surgeons and allied health professionals on a regular basis to focus their energy and intellect on a broad variety of quality improvement initiatives. In this fashion, these groups have turned registry data into information useful for targeted action, thus demonstrating improvement in the process and outcomes of cardiovascular surgical care in each of their regions. 


\section{Abbreviations and Acronyms \\ $\mathrm{CABG}=$ coronary artery bypass graft \\ IMPROVE $=$ Cardiac Surgery Quality Improvement Network \\ $\mathrm{RBC} \quad=$ red blood cell \\ STS $=$ Society of Thoracic Surgeons}

Representatives from several of these regional collaboratives recently convened to discuss opportunities to drive quality assurance and improvement on a much larger scale. Although each collaborative has a similar mission, namely the improvement of cardiothoracic surgical care and outcomes, no formal process has existed to benchmark performance across these groups. The groups have agreed to share aggregated data and expertise to further evaluate and improve the quality, safety, and cost of cardiac surgical care both within and across regions under the umbrella of the Cardiac Surgery Quality Improvement (IMPROVE) Network (http:// www.improvenetwork.org).

Given prior work in and outside of collaboratives, coupled with growing interest on the topic within the surgical community, members of the IMPROVE Network have agreed to use clinical registry data to benchmark red blood cell (RBC) transfusion practices. In particular, the IMPROVE Network seeks to use these data to assess variability in rates of potentially discretionary RBC transfusions. We report our findings from a prospective, observational study of patients undergoing isolated coronary artery bypass graft (CABG) surgery across 56 medical centers in 4 IMPROVE Network regions.

\section{METHODS}

\section{Study Population}

The IMPROVE Network is composed of 5 regional quality improvement collaboratives: The Clinical Outcomes Assessment Program, Maritime Cardiovascular Quality Initiative, Michigan Society of Thoracic and Cardiovascular Surgeons, Northern New England Cardiovascular Disease Study Group, and Providence Health and Services Cardiovascular Disease Study Group. The IMPROVE Network has developed a set of bylaws to govern its work, along with a mission: "to improve the value of cardiovascular surgical care by developing and sharing best practice knowledge, coordinating, undertaking, evaluating, and disseminating quality improvement projects across our member organizations."

We examined patients undergoing isolated, nonemergent CABG surgery at any of 56 medical centers across 4 IMPROVE Network regions between January 1, 2008, and June 30, 2012.

We excluded any case in which a patient was transfused $\geq 3 \mathrm{RBC}$ units during the intra- or postoperative period. After excluding patients with emergent status and those missing data on gender, the final 200 isolated CABG cases in each center were retained for analysis, leaving a final sample of 11,200 patients from 56 centers and 4 regions. Centers with $<200$ cases meeting the criteria were excluded from the analysis. Due to privacy concerns, only aggregate, de-identified data from each center were used.

The University of Michigan's institutional review board deemed that the use of such data was not regulated (HUM00071282).

\section{Data Collection}

All data were collected locally at each medical center and aggregated to means or percentages at the region level by each collaborative using standardized definitions. ${ }^{1}$ Information specific to preoperative care included patient demographics (eg, age, sex, and weight), comorbid disease (eg, diabetes, peripheral vascular disease, hypertension, dialysis, last preoperative serum creatinine, chronic lung disease, and last preoperative hematocrit), medications (eg, aspirin within 5 days of surgery and other antiplatelet agents), cardiac history (eg, cardiovascular disease, heart failure within 2 weeks of surgery, and myocardial infarction), days from cardiac catheterization to CABG surgery, cardiac anatomy and function (eg, left main disease, ejection fraction, and number of diseased vessels), prior CABG surgery, and urgent status. We additionally collected intraoperative factors, including use of off-pump procedure, crossclamp duration, and cardiopulmonary bypass duration.

\section{Statistical Analysis}

Given that 4 medical centers participated simultaneously in 2 regional collaboratives, each center was randomly allocated to contribute data to only 1 collaborative for analysis.

Demographics are presented as the means of all center means within each region. We similarly present the means within each category for categorical variables. Comparisons between regions were made using analysis of variance.

A logistic regression model using aggregated data from each center was used to compute risk-adjusted rates of transfusion for each region. We explored the effect of pre- and intraoperative factors in our modeling. We include in the logistic regression model a class variable corresponding to the region and pre- and intraoperative center characteristics. Pre- and intraoperative center characteristics were chosen via backward selection with the goal of minimizing Akaike information criterion. ${ }^{2}$ The risk-adjusted rate for each region is calculated by fixing each pre- and intraoperative center characteristic at the same level (ie, the overall mean across all regions) and therefore the risk-adjusted rates are adjusted for differences in pre- and intraoperative factors. Observed rates and asymptotic standard errors were computed from the raw data.

For each region, these rates and their $95 \%$ confidence intervals were plotted. Differences between risk-adjusted regional rates were further assessed by testing for the significance of region as a fixed effect in the adjusted model.

We conducted a sensitivity analysis specifically focused on the contribution of the highest transfusion rate region (ie, Region 4) to our overall findings. Specifically, we compared the adjusted transfusion rates with and without Region 4.

Analyses were performed using R 3.0.1 (R Foundation for Statistical Computing, Vienna, Austria) and SAS 9.3 (SAS Institute Inc, Cary, NC).

\section{RESULTS}

Comparisons of pre- and intraoperative characteristics across the 4 regional collaboratives are displayed in Table 1. Clinically relevant differences did not exist exclusive of some noteworthy exceptions. Region 4 had the highest proportion of patients with severe chronic lung disease $(4.9 \%)$. Region 2 had the highest proportion of myocardial infarctions within 7 days $(39.4 \%)$, urgent status $(73.5 \%)$, aspirin use $(97.8 \%)$, and off-pump procedures $(28.1 \%)$. The use of off-pump procedures in Region 2 was predominantly driven by 1 center.

Within our cohort, $25.2 \%$ of patients (2826 out of $11,200)$ received transfusions of 1 or 2 units RBCs. Of these, $9.7 \%$ received only 1 unit, whereas $15.5 \%$ received 
TABLE 1. Characteristics associated with intra- or postoperative transfusions across the 4 regional collaboratives $(N=56$ centers)

\begin{tabular}{|c|c|c|c|c|c|c|}
\hline \multirow[b]{2}{*}{ Variables } & \multirow[b]{2}{*}{ Overall } & \multicolumn{4}{|c|}{ Region } & \multirow[b]{2}{*}{$P$ value } \\
\hline & & 1 & 2 & 3 & 4 & \\
\hline \multicolumn{7}{|l|}{ Preoperative factors } \\
\hline Age $(y)$ & $64.5(1.3)$ & $64.2(2.0)$ & $65.8(1.0)$ & $65(1.3)$ & $64.1(1.1)$ & .024 \\
\hline Male (\%) & $77.9 \pm 4.5$ & $80.4 \pm 4.5$ & $78.4 \pm 1.5$ & $78.2 \pm 5.1$ & $77.3 \pm 4.4$ & .454 \\
\hline Body surface area $\left(\mathrm{m}^{2}\right)$ & $2.1(0.03)$ & $2(0.03)$ & $2.1(0.02)$ & $2.1(0.01)$ & $2(0.03)$ & $<.001$ \\
\hline Cardiovascular disease & $13.5(3.2)$ & $11.8(2.4)$ & $12.5(4.8)$ & $13.1(3.6)$ & $14.1(2.9)$ & .365 \\
\hline PVD & $13.5(4.3)$ & $13.3(5.9)$ & $16.6(3.8)$ & $11.6(3.1)$ & $14(4.3)$ & .142 \\
\hline \multicolumn{7}{|l|}{ Chronic lung disease } \\
\hline Mild & $14.8(6.3)$ & $11.3(5.0)$ & $12.7(2.6)$ & $13.7(4.8)$ & $16.2(7.0)$ & .220 \\
\hline Moderate & $5(3.6)$ & $1.7(1.3)$ & $4.5(5.8)$ & $5.4(5.4)$ & $5.5(2.6)$ & .099 \\
\hline Severe & $3.8(3.2)$ & $2.2(1.8)$ & $3.6(1.6)$ & $2.2(2.2)$ & $4.9(3.6)$ & .024 \\
\hline Diabetes & $40.6(5.0)$ & $40(5.3)$ & $38.6(5.7)$ & $38.6(5.4)$ & $41.9(4.6)$ & .180 \\
\hline Dialysis & $1.5(1.4)$ & $1.9(0.80)$ & $2.8(3.8)$ & $1.4(0.9)$ & $1.3(1.1)$ & .169 \\
\hline \multicolumn{7}{|l|}{ Number of diseased vessels } \\
\hline 0 or 1 & $4.8(2.7)$ & $4.8(2.09)$ & $4(3.1)$ & $5(3.0)$ & $4.7(2.7)$ & .931 \\
\hline 2 & $20.9(5.8)$ & $21.2(6.8)$ & $20.6(5.9)$ & $21.6(7.4)$ & $20.6(5.0)$ & .961 \\
\hline 3 & $74.4(7.3)$ & $74(7.8)$ & $75.4(4.3)$ & $73.4(9.6)$ & $74.7(6.6)$ & .941 \\
\hline \multicolumn{7}{|l|}{ Ejection fraction } \\
\hline$<40 \%$ & $12(3.8)$ & $13.2(4.3)$ & $14(1.9)$ & $10.5(3.3)$ & $12.3(4.0)$ & .261 \\
\hline $40 \%-49 \%$ & $15.2(3.9)$ & $13.5(3.0)$ & $13.9(1.9)$ & $15.3(3.3)$ & $15.7(4.4)$ & .556 \\
\hline $50 \%-59 \%$ & $31.7(7.8)$ & $25.7(5.8)$ & $26.7(6.6)$ & $29.7(5.6)$ & $34.3(8.1)$ & .016 \\
\hline$\geq 60 \%$ & $41(9.7)$ & $47.7(8.1)$ & $45.4(9.6)$ & $44.5(8.3)$ & $37.7(9.5)$ & .021 \\
\hline Heart failure within $2 \mathrm{wk}$ & $11.1(7.6)$ & $11.8(3.7)$ & $10.7(4.3)$ & $14.9(11.3)$ & $9.3(5.8)$ & .155 \\
\hline \multicolumn{7}{|l|}{ Hematocrit } \\
\hline$<36 \%$ & $20.4(5.7)$ & $18.8(4.8)$ & $23.8(4.3)$ & $20.3(5.0)$ & $20.4(6.4)$ & .597 \\
\hline $36 \%-39 \%$ & $29.5(3.7)$ & $31.2(3.5)$ & $31.4(3.8)$ & $28.4(3.7)$ & $29.5(3.8)$ & .321 \\
\hline $40 \%-42 \%$ & $26(3.8)$ & $26.5(4.6)$ & $25.4(2.7)$ & $26(3.9)$ & $26.1(3.9)$ & .978 \\
\hline$\geq 43 \%$ & $24(5.6)$ & $23.6(4.2)$ & $19.4(4.4)$ & $25.3(5.2)$ & $24(6.0)$ & .320 \\
\hline Hypertension & $86.4(6.2)$ & $84.8(5.7)$ & $88.6(3.6)$ & $84(7.5)$ & $87.5(6.5)$ & .252 \\
\hline Left main disease $\geq 50 \%$ & $33.5(5.9)$ & $36.1(6.4)$ & $33.9(5.4)$ & $34.3(4.7)$ & $32.7(6.3)$ & .585 \\
\hline Myocardial infarction & $50.9(7.7)$ & $51.1(11.3)$ & $61.8(8.1)$ & $53.6(5.5)$ & $48.3(13.5)$ & .002 \\
\hline Recent myocardial infarction $(\leq 7 \mathrm{~d})$ & $25.3(7.7)$ & $24.6(6.4)$ & $39.4(7.7)$ & $25(8.3)$ & $23.9(6.1)$ & .001 \\
\hline Urgent status & $59.6(14.6)$ & $47.1(13.3)$ & $73.5(5.9)$ & $54.4(14.3)$ & $62.5(13.5)$ & .008 \\
\hline Redo coronary artery bypass graft & $2.2(1.4)$ & $3.1(1.9)$ & $2(1.4)$ & $1.9(1.2)$ & $2.3(1.4)$ & .389 \\
\hline Days from cardiac catheterization to surgery & $12.3 \pm 6.1$ & $10.9 \pm 5.0$ & $15.9 \pm 10.8$ & $12.9 \pm 5.0$ & $11.9 \pm 6.2$ & .59 \\
\hline \multicolumn{7}{|l|}{ Medication use within $5 \mathrm{~d}$} \\
\hline Antiplatelet agents & $2.9(7.8)$ & $9.5(22.0)$ & $9.3(6.0)$ & $1.1(1.0)$ & $1.6(3.1)$ & .03 \\
\hline Aspirin & $90.9(6.8)$ & $83.0(9.0)$ & $97.8(1.0)$ & $86.7(6.3)$ & $93.5(4.4)$ & $<.001$ \\
\hline Last serum creatinine $(\mathrm{mg} / \mathrm{dL})$ & $1.1(0.1)$ & $1.4(0.08)$ & $1.1(0.5)$ & $1.1(0.1)$ & $1.1(0.1)$ & $<.001$ \\
\hline \multicolumn{7}{|l|}{ Intraoperative factors } \\
\hline Off-pump procedure & $11.5(19.0)$ & $5.1(8.2)$ & $28.1(46.3)$ & $12.2(21.8)$ & $10.2(15.5)$ & .32 \\
\hline Crossclamp duration (min) & $74.3 \pm 17.2$ & $68.7 \pm 18.2$ & $67.3 \pm 8.3$ & $76.8 \pm 18.4$ & $75.2 \pm 17.5$ & .65 \\
\hline Bypass duration $(\% \mathrm{~min})$ & $100.2 \pm 17.0$ & $93.7 \pm 17.1$ & $101.7 \pm 6.4$ & $101.3 \pm 19.6$ & $99.9 \pm 13.0$ & .80 \\
\hline
\end{tabular}

Values are presented as $\mathrm{n}(\%)$ or mean \pm standard deviation. $P V D$, Peripheral vascular disease.

2 units (Figure 1). Significant variation in use and number of RBC units existed across regions (no units, $74.8 \%$ [min-max, 70.0\%-84.1\%], 1 unit, 9.7\% [min-max, 5.1\%-11.8\%], and 2 units, $15.5 \%$ [min-max, $9.1 \%-18.2 \%$ ]; $P<.001$ ) (Table 2). Variation in overall transfusion rates remained after adjusting for preand intraoperative center characteristics $(9.1 \%-31.7 \%$; $P<.001)$, as depicted in Figure 2. These findings were qualitatively unchanged when excluding Region $4(P=.004)$.

\section{DISCUSSION}

Within our cohort, $25.2 \%$ of patients (2826 out of $11,200)$ received transfusions of 1 or 2 units RBC. Rates of 1-unit transfusions as well as 2-unit transfusions varied more than 2-fold across regions. There was a $22.6 \%$ absolute difference $(9.1 \%-31.7 \%)$ in rates across regions after adjustment. These findings suggest that there is a lack of consensus across geographic regions in use of small volumes of RBCs. 


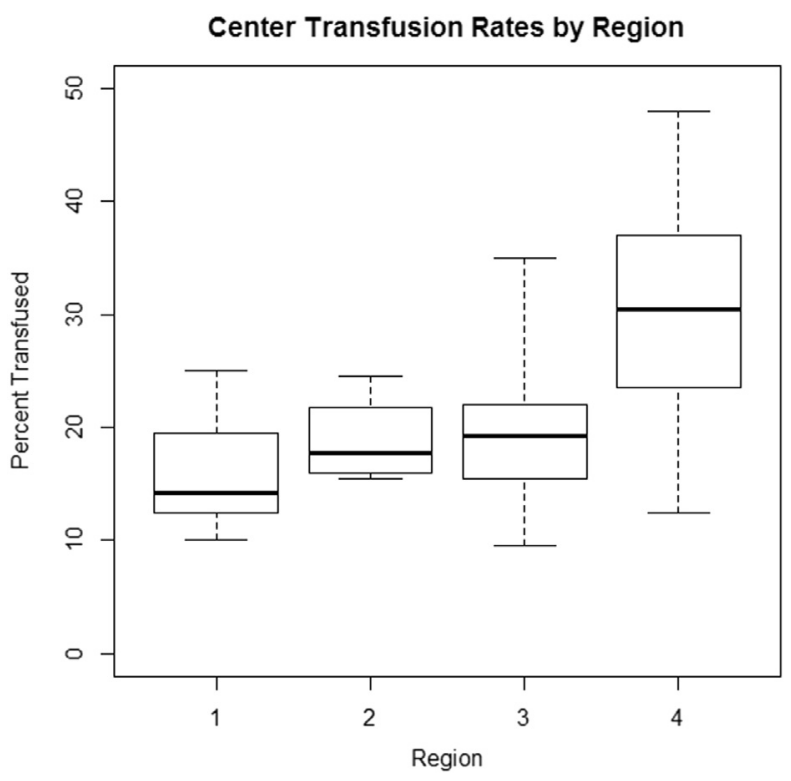

FIGURE 1. Center transfusion rates by region. Boxes represent the interquartile range within each center; whiskers represent the minimum and maximum center-level transfusion rate within each region.

Although in some circumstances blood transfusions are life-preserving (eg, replacement of volume in the setting of acute blood loss), evidence suggests that some transfusions may be associated with harm. ${ }^{3}$ Major bleeding is a rare phenomenon, although 1 that is associated with increased risk of mortality. ${ }^{4}$ To address confounding by indication, some investigators have studied the short- and long-term influence of small units of RBCs (ie, 1-2 units), because these are likely not given to address active bleeding. Parallel work from several investigative teams have found that transfusions of 1 to 2 units RBCs are associated with a $90 \%$ increased odds of operative mortality, ${ }^{5}$ and a $16 \%$ increased hazard of long-term ${ }^{3}$ mortality after cardiac surgery. These compelling data suggest that small units of RBCs may have an important influence on patient outcomes, and identifying determinants of their use is worthy of further investigation. Although not discounting the potential influence of larger volumes of RBCs, we deliberately excluded patients who received RBCs $(>3$ units) that may have been given to address active bleeding.

Beyond acute blood loss, small units of RBC transfusions may be given in cardiac surgery for a number of reasons, including decreased hematocrit levels and poor oxygen carrying capacity. Falling hematocrit levels may occur in patients experiencing hemodilutional anemia during cardiopulmonary bypass. ${ }^{6}$ Clinical practice guidelines may help guide transfusion decisions. ${ }^{7}$ In its most recent guidelines, the STS, in conjunction with the Society of Cardiovascular Anesthesiologists and the International Consortium for Evidence-Based Perfusion, states that transfusions are reasonable when hematocrit is $\leq 18 \%$ (class IIa/level C recommendation). The clinical team must balance the benefits of treating anemia with RBCs against the potential harm associated with anemia and the RBC units themselves. ${ }^{4}$ For this analysis we did not have data from each collaborative concerning hematocrit levels before the transfusion decisions, nor the type of prophylactic strategies that may have been undertaken to prevent anemia, including reduction of circuit prime volume, ultrafiltration, or the use of retrograde autologous priming. ${ }^{7,8}$ As such, our study was unable to determine the extent to which these and other strategies are differentially adopted across regions, or how their use may help explain the apparent variation in transfusion rates.

For some time researchers at the Dartmouth Atlas of Health Care have used Medicare claims data to reveal that the choice of treatment is invariably driven by geographically distinct styles of practice, rather than differences in patient presentation. ${ }^{9}$ Additionally, others have found that practice patterns may also be driven by factors at the provider level. For instance, Salem-Schatz and colleagues ${ }^{10}$ found that a physician's knowledge concerning clinical indications for transfusions as well as his/her receptivity to input from colleagues may influence the appropriateness of transfusions. A recent survey of cardiac team members suggests that only $20 \%$ had institutional discussions concerning the STS blood management guidelines, whereas only $14 \%$ had formed institutionally based working groups. ${ }^{11}$ Jin and colleagues ${ }^{12}$ reported a prospective study across 12 hospitals participating in the Providence Health and Services Cardiovascular Disease Study Group, in which they sought to disentangle physician and institutional contributors to transfusion decisions. They found that the variance of transfusions across institutions was more than twice that of surgeons practicing within a facility; surgeons contributed to $30 \%$ of the variation in transfusion practice, whereas the institution contributed the remaining $70 \%$.

TABLE 2. Observed transfusion of 1 or 2 units red blood cells, by region

\begin{tabular}{lccccc}
\hline & & \multicolumn{3}{c}{ Region } \\
\cline { 3 - 6 } Number of units transfused & Overall & $\mathbf{1}$ & $\mathbf{2}$ & $\mathbf{3}$ & $\mathbf{4}$ \\
\hline 0 & 74.8 & $84.1(75.0-90.0)$ & $81.1(75.5-84.5)$ & $80.0(65.0-90.5)$ & $70.0(52.0-87.5)$ \\
1 & 9.7 & $5.1(2.5-11.0)$ & $9.8(5.0-15.0)$ & $6.8(1.0-22.5)$ & $11.8(2.5-24.0)$ \\
2 & 15.5 & $10.8(7.5-19.0)$ & $9.1(2.5-11.0)$ & $13.3(6.5-19.5)$ & $18.2(4.0-29.5)$ \\
\hline
\end{tabular}

Values are presented as mean across centers (minimum-maximum center values) within each region. 


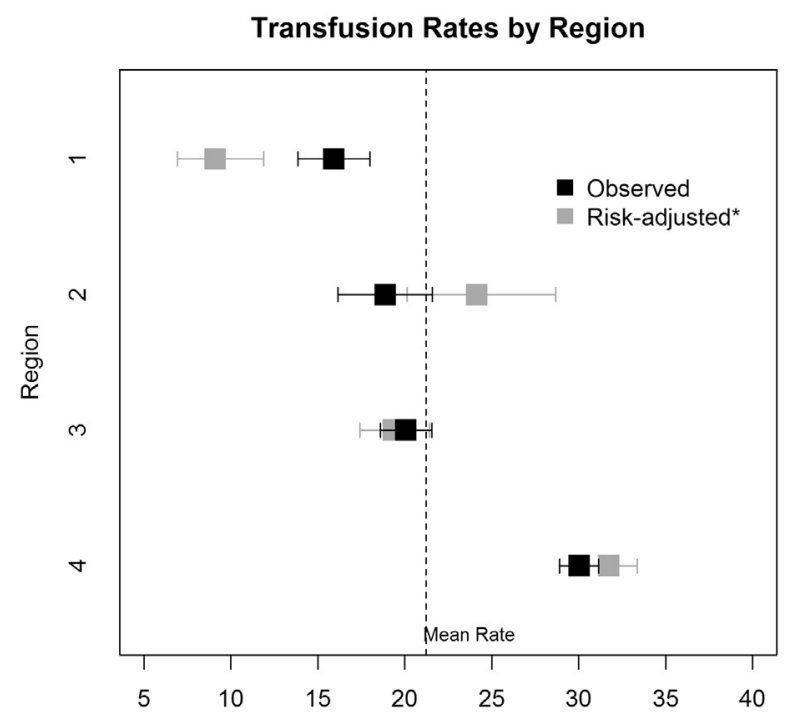

FIGURE 2. Transfusion rates by region. Black boxes represent observed rates of 1 to 2 units of red blood cell transfusions, whereas gray boxes represent risk-adjusted rates. Error bars represent $95 \%$ confidence intervals for observed and risk-adjusted rates. *Adjusted for age, sex, body surface area, hematocrit, dialysis, creatinine, 3-vessel disease, crossclamp duration, bypass duration, antiplatelet agents, and aspirin use.

From this the authors concluded that hospitals, rather than individual surgeons, predominantly drive transfusion practice. Although not restricted to small volumes of RBCs, the work by Jin and colleagues ${ }^{12}$ suggests that hospitals have their own transfusion signature. Our finding complements this growing body of literature by suggesting that geographic regions themselves may have their own transfusion signature. Within cardiac surgery, these regions are represented by quality improvement collaboratives whose members meet internally to discuss quality and outcomes and define practices to drive quality improvement. Until recently these regional collaboratives have not had a forum to share benchmarking data with each other. Ideally, the partnering of the collaboratives through the IMPROVE Network will be a means for sharing benchmarking data to drive quality improvement on a larger geographic scale.

Our finding of geographic variability in RBC transfusion rates is supported by previous studies. Using national STS data, Bennett-Guerrero and colleagues ${ }^{13}$ observed geographic differences in RBC (as well as other blood products) use at 798 institutions performing 102,470 primary isolated CABG procedures. Use was highest $(61 \%)$ in the West South Central region (ie, Oklahoma, Louisiana, Arkansas, and Texas) of the United States, whereas lowest (50\%) in the Mountain region (ie, Arizona, Colorado, Idaho, Montana, Nevada, New Mexico, Utah, and Wyoming). Similarly, wide variability occurred in rates of transfusion of RBCs and other blood products among patients undergoing $\mathrm{CABG}$ surgery with cardiopulmonary bypass in US hospitals. Maddux and colleagues ${ }^{14}$ used a dataset from a perfusion services provider to document rates of RBC transfusions across 17,252 isolated CABG procedures conducted at 1 of any 144 institutions located in 32 states and Puerto Rico. Those authors reported transfusion rates of $40.8 \%$ across institutions, although the rates varied (Midwest, 33.7\%; South, 36.1\%; West, $40.5 \%$; and Northeast, $43.1 \%$ ). Together, these reports suggest that a patient's risk of transfusion may in part be driven by factors other than patient presentation, including the practice patterns of the region in which they seek care.

As is the case with any observational study, our findings are subject to confounding at the level of the patient, provider, medical center, and region. Although we adjusted for many common risk factors for blood transfusions, including preoperative hematocrit, lingering confounding may exist, including the reasons underlying the transfusion decision. Although we are not able to fully rule out the influence of unmeasured confounding, we have made efforts to address these confounding factors both in our study design and analysis, including limiting our cohort to patients receiving only small increments of blood transfusions and undergoing nonemergent operations, and performing multivariable logistic regression analyses. We used center-level aggregate data to describe and adjust for differences in pre- or intraoperative practices. These data may in some circumstances not appropriately reflect variation at the patient or center level. Last, we hypothesize that apparent regional differences in transfusion practices across the IMPROVE Network may in part be explained by regional supply and demand of blood products, or more (or less) intensive marketing efforts by agencies (eg, American Red Cross) to limit potentially unnecessary transfusion practices. Although we were unable to account for these regional effects, we anticipate that they would have a marginal effect, although they would drive our findings toward null.

Our findings suggest that there is wide geographic variability in the use of potentially discretionary RBC transfusions. Our findings persisted even after adjusting for known factors that may influence transfusion rates. Further investigation is warranted to improve our understanding of why such variability in transfusion rates persists. Partnerships across geographic regions, including through the IMPROVE Network, may serve as a vehicle for undertaking and evaluating the effectiveness of such investigations, including those seeking to reduce unwarranted variability in practice.

\section{References}

1. Society of Thoracic Surgeons. STS adult cardiac database data specifications. Version 2.73. Chicago: Society of Thoracic Surgeons; 2011. Available at: http://www.sts.org/sites/default/files/documents/word/STSAdultCVDataSpecifi cationsV2_73\%20with\%20correction.pdf. 
2. Agresti A. Categorical data analysis. New York: Wiley-Interscience; 2002.

3. Surgenor SD, Kramer RS, Olmstead EM, Ross CS, Sellke FW, Likosky DS, et al. The association of perioperative red blood cell transfusions and decreased longterm survival after cardiac surgery. Anesth Analg. 2009;108:1741-6.

4. Ranucci M, Baryshnikova E, Castelvecchio S, Pelissero G. Major bleeding, transfusions, and anemia: the deadly triad of cardiac surgery. Ann Thorac Surg. 2013;96:478-85.

5. Paone G, Likosky DS, Brewer R, Theurer PF, Bell GF, Cogan CM, et al, Transfusion of 1 and 2 units of red blood cells is associated with increased morbidity and mortality. Ann Thorac Surg. 2014;97:87-93; discussion 94.

6. Surgenor SD, DeFoe GR, Fillinger MP, Likosky DS, Groom RC, Clark C, et al. Intraoperative red blood cell transfusion during coronary artery bypass graft surgery increases the risk of postoperative low-output heart failure. Circulation. 2006;114:I43-8.

7. Ferraris VA, Brown JR, Despotis GJ, Hammon JW, Reece TB, Saha SP, et al. 2011 update to the Society of Thoracic Surgeons and the Society of Cardiovascular Anesthesiologists blood conservation clinical practice guidelines. Ann Thorac Surg. 2011;91:944-82.

8. Shann KG, Likosky DS, Murkin JM, Baker RA, Baribeau YR, DeFoe GR, et al. An evidence-based review of the practice of cardiopulmonary bypass in adults: a focus on neurologic injury, glycemic control, hemodilution, and the inflammatory response. J Thorac Cardiovasc Surg. 2006;132:283-90.

9. Dartmouth Medical School, Center for the Evaluative Clinical Sciences. The Dartmouth atlas of health care, 1998. Chicago: American Hospital Publishing; 1998.

10. Salem-Schatz SR, Avorn J, Soumerai SB. Influence of knowledge and attitudes on the quality of physicians' transfusion practice. Med Care. 1993;31:868-78.

11. Likosky DS, FitzGerald DC, Groom RC, Jones DK, Baker RA, Shann KG, et al Effect of the perioperative blood transfusion and blood conservation in cardiac surgery clinical practice guidelines of the Society of Thoracic Surgeons and the Society of Cardiovascular Anesthesiologists upon clinical practices. Anesth Analg. 2010;111:316-23.

12. Jin R, Zelinka ES, McDonald J, Byrnes T, Grunkemeier GL, Brevig J. Effect of hospital culture on blood transfusion in cardiac procedures. Ann Thorac Surg. 2013;95:1269-74.

13. Bennett-Guerrero E, Zhao Y, O'Brien SM, Ferguson TB Jr, Peterson ED, Gammie JS, et al. Variation in use of blood transfusion in coronary artery bypass graft surgery. JAMA. 2010;304:1568-75.

14. Maddux FW, Dickinson TA, Rilla D, Kamienski RW, Saha SP, Eales F, et al. Institutional variability of intraoperative red blood cell utilization in coronary artery bypass graft surgery. Am J Med Qual. 2009;24:403-11.

\title{
EDITORIAL COMMENTARY
}

\section{Blood transfusion balance: Too much, not enough, or just right}

\author{
Victor A. Ferraris, MD, PhD
}

See related article on pages 3084-9.

The decision to administer a perioperative blood transfusion involves a balance between the risks of receiving an allogeneic donor unit and the benefits of volume replacement and improved oxygen-carrying capacity. Every experienced surgeon has a patient whose life was saved by a blood transfusion. On the other hand, an overwhelming amount of evidence suggests that perioperative blood transfusion translates into adverse surgical outcomes in some circumstances. In their study published in this issue of the Journal, Likosky and coauthors ${ }^{1}$ evaluated a subgroup of patients undergoing elective coronary artery bypass grafting (CABG) who received a so-called discretionary transfusion of packed red blood cells (PRBCs). They used a subset of the Society of Thoracic Surgeons adult cardiac surgical database from

\footnotetext{
From the Department of Surgery, University of Kentucky, Lexington, Ky.

Disclosures: Author has nothing to disclose with regard to commercial support.

Received for publication Sept 19, 2014; accepted for publication Sept 20, 2014; available ahead of print Oct 16, 2014.

Address for reprints: Victor A. Ferraris, MD, PhD, Tyler Gill Professor of Surgery, University of Kentucky, A301 Kentucky Clinic, 740 South Limestone, Lexington, KY 40536-0284 (E-mail: Ferraris@earthlink.net).

J Thorac Cardiovasc Surg 2014;148:3089-91

$0022-5223 / \$ 36.00$

Copyright (C) 2014 by The American Association for Thoracic Surgery

http://dx.doi.org/10.1016/j.jtcvs.2014.09.071
}

5 regional collaboratives in the Cardiac Surgery Quality Improvement (IMPROVE) network to identify 2 groups of patients: 1 group receiving 1 or 2 units of PRBCs and 1 with no transfusions. The study cohort was at low risk of operative mortality, but independent of this risk there was significant variation in the transfusion of 1 or 2 PRBC units across regions.

Patients with low operative risk are least likely to benefit from transfusion. For trauma patients, the association of transfusion with all-cause mortality appears to vary according to the predicted risk of death. Transfusion may reduce mortality in patients at high predicted risk of death but increase mortality in those at low risk (Figure 1). ${ }^{2}$

Similarly, we found a dose-dependent relationship between blood transfusion and morbidity and mortality in patients undergoing noncardiac thoracic operations. ${ }^{3}$ Further, we queried the American College of Surgeons National Surgical Quality Improvement Program database, which contains patients undergoing mostly general surgical procedures, for data entered between 2010 and 2012 to identify mortality and morbidity differences in patients receiving blood transfusion within 72 hours of the operative procedure and those who did not receive any blood. We calculated the relative risk of development of a serious complication or of operative mortality in propensity-matched patients with equivalent risks of receiving a blood transfusion. There was an astounding 